\title{
Avaliação do Autoconceito no Contexto Escolar: Análise das Publicações em Periódicos Brasileiros
}

Assessment of self-concept in the school context: Analysis of the publications in brazilian journals

Evaluación del auto concepto en el contexto escolar: Análisis de las publicaciones en periódicos brasileños

Adriana Cristina

Boulhoça Suehiro, Fabián Javier Marín Rueda, Evelin Zago de Oliveira \& Silvia Verônica Pacanaro

Universidade São Francisco/Itatiba 
Resumo: Visando a levantar as publicações dos últimos dez anos referentes à avaliação do autoconceito no contexto escolar, o presente estudo avaliou artigos encontrados nos periódicos classificados como Nacional A pela CAPES. Os dados evidenciaram poucas publicações voltadas para a avaliação do construto. Mesmo os periódicos específicos de Psicologia escolar e educacional passaram a publicar artigos sobre o assunto somente a partir de 2005. Nas análises por Região, autoria e amostras, o Sudeste apresentou maior concentração de artigos publicados. Foi identificado o uso mais freqüente da autoria múltipla e a realização de estudos envolvendo crianças. As referências apresentaram mais artigos e livros, com predominância da literatura internacional. Quanto aos instrumentos de avaliação, os mais utilizados foram a Escala Infantil Piers-Harris e a Escala de Autoconceito Infanto-Juvenil EAC-IJ. Por fim, o presente estudo atesta a necessidade de novas publicações voltadas para a avaliação do autoconceito no contexto escolar, guiado pela importância do construto no processo de aprendizagem.

Palavras-chave: Avaliação psicológica. Autoconceito. Contexto escolar. Produção científica.

Abstract: Aiming to do research on the publications of the last ten years, regarding the evaluation of the self-concept in the school context, the present study evaluated the articles found in journals classified as Nacional $A$ by CAPES. The data showed that few publications were related to this subject, and even the education journals only started to publish on the subject from 2005 on. Among the analyzes concerning the area, authorship and samples, the Southeast presented a larger concentration of articles. It was identified the frequent use of the multiple authorship and research on children. The references presented more articles and books, with predominance of the international literature. As for the evaluation instruments, the most used were the Piers-Harris Children Scale and the Children and Youth Self-Concept Scale EAC-IJ. Finally, the present study indicated the need of new publications regarding the evaluation of the self-concept in the school context, guided by the importance of the construct in the learning process.

Keywords: Psychological assessment. Self-concept. School context. Scientific production.

Resumen: Con el objetivo de reunir las publicaciones de los últimos diez años referentes a la evaluación del auto concepto en el contexto escolar, el presente estudio evaluó artículos encontrados en los periódicos clasificados como Nacional A por la CAPES. Los datos evidenciaron pocas publicaciones devotadas a la evaluación del constructo. Incluso los periódicos específicos de Psicología escolar y educacional pasaron a publicar artículos sobre el asunto solamente desde 2005. En los análisis por Región, autoría y muestras, el Sudeste presentó mayor concentración de artículos publicados. Fue identificado el uso más frecuente de la autoría múltiple y la realización de estudios involucrando niños. Las informes presentaron más artículos y libros, con predominancia de la literatura internacional. En cuanto a los instrumentos de evaluación, los más utilizados fueron la Escala Infantil Piers-Harris y la Escala de Auto concepto Infanto-Juvenil EAC-IJ. Por fin, el presente estudio testifica la necesidad de nuevas publicaciones devotadas para la evaluación del auto concepto en el contexto escolar, guiado por la importancia del constructo en el proceso de aprendizaje.

Palabras clave: Evaluación psicológica, Auto concepto. Contexto escolar. Producción científica.

O autoconceito pode ser definido como uma atitude que o indivíduo tem em relação a si mesmo, decorrente da maneira como se percebe (Oliveira, 1994). Para Teixeira e Giacomini (2002), o autoconceito incorpora, além de crenças percebidas sobre a competência individual em situações específicas, crenças de valor sobre si mesmo. Embora o construto autoconceito seja explorado por diversos enfoques psicológicos, alguns aspectos carecem ainda de melhor esclarecimento. Para Burns (1979), o autoconceito possui três componentes básicos, a saber, um componente cognitivo, um comportamental e um afetivo. O componente cognitivo se refere ao conjunto de características com o qual a pessoa se descreve e que não necessariamente deve ser verdadeiro ou objetivo, mas orientaria seu modo habitual de ser e de se comportar. $\mathrm{O}$ aspecto comportamental é influenciado diretamente pelo conceito que a pessoa tem de si mesma, e, por último, o componente afetivo, que diz respeito aos afetos e emoções que acompanham a descrição de si mesmo, e que foi definida por Coopersmith (1967) como auto-estima. 
Segundo Hugles (1984) e Marsh (1993), o termo auto-estima muitas vezes é encontrado na literatura como sinônimo de autoconceito. No entanto, o autoconceito seria um construto mais amplo, que incluiria os aspectos cognitivos, afetivos e comportamentais, enquanto a auto-estima seria um componente mais avaliativo e limitado do que o autoconceito.

Há um consenso na literatura de que o autoconceito começa a ser construído na infância. Assim, não se nasceria com um conceito próprio, predeterminado, mas ele se desenvolveria no decorrer da vida. Coopersmith (1967) afirmou que as crianças recebem informações sobre si mesmas dos adultos significativos em sua vida, e que, com base nessas impressões recebidas, desenvolvem uma imagem positiva ou negativa sobre si mesmas.

A partir dessa idéia de Coopersmith, muitos autores buscaram ampliar os conhecimentos relacionados ao desenvolvimento do autoconceito. Dessa forma, dentre os modelos teóricos propostos, o de Shavelson, Hubner e Stanton (1976) passou a ser o mais aceito a partir da década de 80 do século passado. Esse modelo trabalha com o autoconceito de forma hierárquica e multifacetária. Logo, o autoconceito seria a percepção que o indivíduo tem de si, sustentado diretamente por suas experiências em relação aos outros e pela valoração que a pessoa faz de sua própria conduta. O modelo traz a proposta de um autoconceito geral, que inclui autoconceitos mais concretos e específicos. Assim, existiriam os componentes afetivos, que seriam mais subjetivos e internos; os sociais, que estariam mais relacionados com o significado que a conduta dos indivíduos tem para os demais; os físicos, que seriam fundamentais nas atitudes e na aparência geral dos indivíduos, e os acadêmicos, que dizem respeito ao sentimento que a criança tem perante seu contexto escolar e que, na maioria das vezes, é formado pela interação com os colegas e professores.

Para Shavelson et al. (1976), esses quatro componentes representariam o nível secundário, ou seja, seriam considerados como a parte interna de uma representação hierárquica do autoconceito, sendo que acima deles se encontra o autoconceito global ou geral. Como já mencionado, foi a partir do final do século passado que o modelo dos autores citados ganhou força e começou a ser pesquisado. Nesse sentido, pesquisas verificaram a relação do autoconceito com o meio social (Blascovich \& Tomaka, 1991; Harter, 1982), com a família (Borrine, 1991), com a raça (Lay \& Wakstein, 1985; Osborne \& Legette, 1982) e com a idade (Crain \& Bracken, 1994; Marsh, 1989; Walker \& Greene, 1986).

No Brasil, observa-se, nas dissertações e teses, uma gama variada de pesquisas referentes ao autoconceito em diferentes contextos bem como sua relação com outras variáveis que focalizam o construto geral. Um exemplo dessa relação é o estudo de Ribeiro (1987), que pesquisou um grupo de adolescentes levando em consideração também a estrutura familiar. Outros apontam a importância da atividade física para a construção e desenvolvimento do autoconceito em crianças (Chiapeta, 1988; Palma, 1996). Já Costa (1996) pesquisou sua influência na satisfação no trabalho de bancários, verificando uma estreita relação entre eles. Por sua vez, Monteiro (1996) investigou a influência das mensagens dos meios de comunicação no autoconceito e na auto-imagem em jogadores de futebol. Ainda Giavonni $(1997,2000)$ estudou o construto autoconceito, construindo e validando um 
inventário do self. Por fim, Valentini (1999) procurou avaliar o significado do corpo para adolescentes de ambos os sexos e relacioná-lo com o autoconceito dos mesmos.

Como apresentado, o autoconceito tem sido investigado por pesquisadores de diversas áreas do conhecimento, especialmente pela Psicologia. Embora já estudado em vários âmbitos, aqui ele será centrado nas formas pelas quais tem sido avaliado no contexto educacional. Tal enfoque se deve à importância da integração dos fatores cognitivos e sociais no sucesso da criança nesse contexto, pois é no momento em que ela entra na escola que percebe o quanto é capaz de realizar e satisfazer as expectativas dos pais e educadores. Portanto, é ao longo das etapas de escolarização que a criança vai se tornando consciente do quanto ela é capaz de realizar e aprender. Dessa forma, experimentar o fracasso escolar poderá marcar para sempre sua vida (Sisto \& Martinelli, 2004).

Medeiros (2000) evidenciou a existência de relação entre o autoconceito e o rendimento escolar e a resolução de problemas.
Nesse sentido, Simões (1997) afirma que o autoconceito escolar pode ser definido como o universo de representações que o aluno tem das suas capacidades e realizações escolares bem como das avaliações que ele faz dessas capacidades e realizações. Por sua vez, Stein (1996) relata que o termo autoconceito escolar tem sido caracterizado pela utilização de dois elementos comuns à maioria das pesquisas. Em alguns casos, o autoconceito escolar tem sido estudado tanto a partir de uma reflexão descritiva ("Eu gosto de matemática") como de aspectos avaliativos de sua percepção ("Eu sou bom em matemática"). Num segundo caso, a sua autopercepção associada a seu autoconceito acadêmico tende a focalizar-se na competência como estudante, mais que sobre sua atitude.
Estudos que enfocam as relações entre o autoconceito e a escola foram realizados, no exterior, desde a década de 70 do século passado. Nesse sentido, há concordância na literatura de que o autoconceito seria um dos fatores principais do rendimento acadêmico (Buchanan \& Wolf, 1986; Carroll, Friedrich, \& Hund, 1984; McGuire, 1987; Purkey, 1970), o que também tem sido focalizado em algumas pesquisas realizadas no Brasil (Amorim, 2000; Castro, 1999; Jacob \& Loureiro, 1999; Jacob, 2001; Jesus \& Gama, 1991; Suehiro, 2006).

No território nacional, essas relações foram temas de teses e dissertações, como a de Jeronymo (2003), que trabalhou com crianças de pais alcoólatras e não alcoólatras, em função do seu desempenho e seu autoconceito. Lima (1996) pesquisou se o fracasso escolar poderia influenciar no baixo autoconceito de adolescentes que freqüentaram ou freqüentam o ensino noturno. Nessa mesma linha, Martins (1997) averiguou diferenças no autoconceito de crianças repetentes e não repetentes de ambos os sexos. Já Silva (1999) investigou as relações entre o autoconceito e o rendimento acadêmico de adolescentes, percebendo que o autoconceito seria um fator determinante na auto-avaliação do aluno.

Estudando alunos com alto desempenho acadêmico, Castro (1999) verificou que eles tiveram também um escore elevado de autoconceito acadêmico e maiores habilidades metacognitivas em relação ao conhecimento e ao uso de estratégias reguladoras do próprio conhecimento. Por sua vez, Medeiros (2000) evidenciou a existência de relação entre o autoconceito e o rendimento escolar e a resolução de problemas. 
Como verificado, as pesquisas de autoconceito podem ser encontradas numa quantidade consideravelmente grande nas teses e dissertações do Brasil. Todavia, no âmbito escolar, ainda há poucos estudos sobre a avaliação do construto, o que pode ser explicado pela carência de formas válidas de medida. Deve-se levar em consideração, ainda, que existe um grande volume de pesquisas que estão sendo realizadas em todas as áreas da ciência que, na maioria das vezes, permanecem somente no âmbito acadêmico, como literatura "cinzenta". Tal fato acaba por dificultar o acesso a esses materiais, seja pela dificuldade de sua inclusão em bases de dados, seja pelo custo elevado de permuta de um material de grandes proporções, especialmente no que se refere ao seu tamanho (Witter, 1999).

Entre os diversos meios de disseminação científica, os periódicos científicos surgem como uma forma eficiente de superação dos problemas de recuperação e divulgação enfrentados com a literatura "cinzenta", possibilitando, assim, a publicação de resultados de pesquisa em todas as áreas de conhecimento (Volpato \& Freitas, 2003). Independentemente do meio de divulgação, considera-se que a produção científica promova crescimento, uma vez que sua divulgação facilita o acesso ao conhecimento em diversas áreas do conhecimento (Freitas, 1998).

Tendo em vista a importância da produção científica, não apenas no Brasil, mas no mundo todo, a análise desse tipo de produção tem se tornado cada vez mais constante. Na Psicologia, a análise da produção científica vem crescendo substancialmente (Yamamoto, Souza, \& Yamamoto, 1999). Em um levantamento da produção científica em periódicos brasileiros entre 1990-1997, Yamamoto et al. (1999) analisaram seis periódicos de circulação nacional especializados da área. Foram encontrados 749 artigos distribuídos pelas 80 edições dos periódicos. Os principais resultados revelaram que as publicações estão concentradas em instituições nas regiões Sul e Sudeste, e que as universidades públicas são as mais produtivas. Ao lado disso, os autores estabeleceram uma relação entre a quantidade de artigos publicados e a existência de programas de pós-graduação nessas universidades. O mesmo foi realizado na pesquisa de Gargantini e Oliveira (2003), que buscou descrever e analisar a produção de estratégias educacionais veiculada no periódico Journal of Educational Psychology, entre 2001 e 2002.

Nesse mesmo contexto, a pesquisa de Oliveira, Cantalice, Joly e Santos (2006) procurou analisar as publicações dos últimos dez anos da revista Psicologia Escolar e Educacional. Os resultados evidenciaram que houve um aumento de artigos publicados nos últimos três anos, sendo que a Região Sudeste foi a que apresentou maior número de trabalhos publicados. Também se pode constatar a predominância da autoria múltipla.

Resultados semelhantes quanto à região de maior produção e à autoria foram obtidos no estudo acerca dos testes psicológicos realizado por Souza-Filho, Belo e Gouveia (2006), no qual foram analisadas 18 revistas e 1182 artigos publicados entre os anos 2000 e 2004. Os resultados indicaram que houve maior concentração das produções na Região Sudeste, e que a autoria múltipla predominou. Ao lado disso, os autores constataram que os artigos que não utilizaram nenhum instrumento psicológico foram os mais freqüentes, e que as universidades mais produtivas na área são aquelas que têm um 
histórico pautado pelo interesse na área da avaliação psicológica, destacando-se entre as primeiras a Universidade São Francisco (USF), a Universidade de Brasília e a Universidade de São Paulo. Diante do panorama encontrado, Souza-Filho et al. (2006) concluíram que a utilização dos testes psicológicos ainda é restrita ao meio acadêmico, uma vez que esse tipo de população ainda é a que está mais intensamente dedicada aos estudos dos instrumentos.

Embora haja divergência entre os diversos autores quanto à adequação do modelo em retratar a realidade editorial do País, assim como discordâncias em relação aos critérios adotados e ao reconhecimento da melhoria das revistas na Psicologia, a avaliação de periódicos científicos continua a ser, de acordo com Costa (2006), o principal instrumento para garantir um padrão mínimo de qualidade nas publicações na área. Dentro desse contexto, o objetivo do presente trabalho foi fazer um levantamento dos trabalhos publicados nos últimos dez anos em periódicos científicos referentes à avaliação do autoconceito no contexto escolar.

\section{Método}

Amostra

Em um primeiro momento, delimitouse a amostra aos periódicos científicos classificados como Nacional $A$ na avaliação realizada pela CAPES/ANPEPP em 2005. Do total de periódicos com essa classificação, apenas se teve acesso a 19 deles, que estavam disponíveis nas bases de dados Scielo e Pepsic. Sendo que artigos que apresentavam o termo "autoconceito" no título ou nas palavras-chave foram encontrados em 10 periódicos. Posteriormente, dos 23 artigos encontrados, trabalhou-se apenas com aqueles que se referiam à avaliação do autoconceito no contexto escolar. Dessa forma, o estudo foi conduzido com um total de 10 artigos científicos encontrados em 6 periódicos científicos, quais sejam, Interação em Psicologia, Paidéia, Psico-USF, Psicologia em Estudo, Psicologia Escolar e Educacional e Psicologia: Reflexão e Crítica. Vale ressaltar que, em todos os casos, pesquisou-se a publicação da última década, ou seja, de 1996 a 2005, porém as revistas Interação em Psicologia e Psicologia em Estudo foram pesquisadas a partir de 1997, ano da sua criação.

\section{Procedimentos}

Os artigos foram analisados na íntegra. Para tanto, foram consideradas as seguintes categorias: autoria, Região em que prevaleceram as pesquisas, instituição de filiação, tipo de população pesquisada, instrumentos utilizados na avaliação do autoconceito e referências utilizadas na fundamentação dos artigos.

O levantamento de todos os dados foi realizado pelos autores da pesquisa, separada e concomitantemente, para dar confiabilidade à avaliação. $\mathrm{O}$ índice de concordância, verificado pela correlação de Pearson, ficou acima dos $85 \%$.

\section{Resultados e discussão}

Inicialmente, foram levantadas as freqüências de publicação de artigos que investigaram a avaliação do autoconceito no contexto escolar e educacional, assim como o ano e o periódico de publicação desses artigos. Verificou-se que somente 10 artigos focalizavam a avaliação do autoconceito no âmbito escolar, sendo que apenas as revistas Psicologia Escolar e Educacional e Psicologia: Reflexão e Crítica 
publicaram mais de um artigo sobre essa temática específica na última década, a saber, quatro e dois artigos, respectivamente.

Observou-se que a média de publicações de artigos referentes à avaliação do autoconceito no contexto educacional é de um artigo por ano, porém, apenas a partir do ano de 2002 é que esse construto começou a ser pesquisado no contexto específico tratado nesta investigação, a saber, a escola. Já na revista Psicologia Escolar e Educacional, foram encontrados artigos sobre esse construto a partir de 2005, o que indica que ele não foi muito pesquisado no campo psicológico, tendo em vista que esse periódico visa à apresentação e à divulgação de pesquisas no campo da Psicologia escolar e educacional e em áreas correlatas.

Esses resultados confirmam aspectos importantes levantados na introdução, quais sejam, a falta de pesquisas voltadas para a avaliação do autoconceito no contexto escolar e a superioridade de trabalhos a respeito do construto na literatura cinzenta, o que restringe o conhecimento do autoconceito e de suas inter-relações somente ao meio acadêmico. Esse fato é ressaltado, por exemplo, nos estudos de Freitas (1998) e Witter (1999), como um obstáculo para as publicações em periódicos científicos.

\section{Autoria}

Com base nos artigos encontrados, procurouse verificar os dados relativos à autoria dos artigos (individual ou múltipla) bem como a Região e a instituição de filiação. Os dados obtidos com relação à autoria mostraram uma prevalência da autoria múltipla. Do total de autores $(N=20)$, cinco eram filiados à Universidade de São Paulo (campus de Ribeirão Preto), sendo que três deles foram autores de dois artigos. O mesmo foi evidenciado nos três autores filiados à Universidade de Brasília, na qual um autor também foi identificado em dois artigos. Da Universidade São Francisco (campus Itatiba) o mesmo autor publicou três artigos diferentes, sendo que mais cinco autores foram identificados como filiados dessa mesma instituição. Da Universidade Estadual de Campinas (UNICAMP), foi evidenciado um mesmo autor em dois diferentes artigos. Por sua vez, dois autores pertenciam à Universidade do Porto (Portugal) e à Universidade de Salamanca (Espanha), e um à Universidade de Mar del Plata (Argentina). Do Centro Universitário Salesiano de Americana (São Paulo), foi identificado apenas um autor.

Com base nesses resultados, percebe-se que as publicações sobre a avaliação do autoconceito no contexto escolar ficaram restritas a apenas duas Regiões, Sudeste, com o total de $60 \%$, e Centro-Oeste, com $20 \%$. Resultado semelhante foi encontrado nas pesquisas de Yamamoto et al. (1999), Gargantini e Oliveira (2003), Souza-Filho et al. (2006) e Oliveira et al. (2006).

Observou-se, ainda, que 20\% dos autores são estrangeiros, o que atesta o desenvolvimento de pesquisas voltadas para a avaliação do construto no exterior. Já no Brasil, como verificado anteriormente, as pesquisas voltadas para esse tema são ainda muito escassas. Isso pode explicar o interesse dos pesquisadores estrangeiros em divulgar seus estudos em periódicos brasileiros. Os resultados obtidos com relação à população pesquisada são apresentados a seguir.

População pesquisada

Em relação à população pesquisada, verificouse que $50 \%$ dos artigos referiram-se somente a 
crianças, enquanto apenas 10\% (um artigo) trabalharam com amostra de crianças e adolescentes, e $40 \%$ foram correspondentes a adolescentes e adolescentes e adultos. Pode-se justificar que o construto estudado ainda pode ser um tema muitas vezes associado somente às crianças. No entanto, estudos com outras faixas etárias seriam necessários não só para aprofundar o estudo da avaliação do autoconceito no âmbito educacional mas também para incentivar a construção e a validação de instrumentos destinados a essas faixas etárias. Esses resultados podem ser observados na literatura, na qual, dentre as pesquisas destacadas referentes ao contexto escolar, a população que recebeu maior destaque foi a infantil (Jeronymo, 2003; Martins, 1997). Na seqüência, são descritos os instrumentos de medida utilizados nos artigos avaliados.

Instrumentos utilizados

Conforme citado anteriormente, os instrumentos de medida utilizados nos artigos avaliados também foram objeto de investigação deste estudo. Exploraram-se os resultados referentes à freqüência dos instrumentos de avaliação sobre o autoconceito utilizados no contexto educacional. Esses resultados podem ser observados na Tabela 1.

Tabela 1. Freqüência de instrumentos empregados na avaliação do autoconceito no contexto escolar pelos artigos focalizados.

\begin{tabular}{lll}
\hline Instrumentos de avaliação do autoconceito & F & (\%) \\
\hline Escala Infantil Piers - Harris de Autoconceito & 2 & 20 \\
Escala de Autoconceito Infanto-Juvenil EAC-IJ & 2 & 20 \\
Teste de Autoconceito - Forma A & 1 & 10 \\
Escala de Autoconceito Estatístico & 1 & 10 \\
Escala de Perfil de Autopercepção para Crianças & 1 & 10 \\
Escala de Avaliação de Autoconceito & 1 & 10 \\
Escala Fatorial de Autoconceito - EFA & 1 & 10 \\
Self - Description Questionnaire III & 1 & 10 \\
Total & 10 & 100 \\
\hline
\end{tabular}

Sob essa perspectiva, os instrumentos mais utilizados para a avaliação do autoconceito neste contexto foram a Escala Infantil Piers - Harris de Autoconceito e a Escala de Autoconceito InfantoJuvenil EAC-IJ correspondente a $40 \%$ do total da amostra. Deve-se ressaltar que foi considerado que, em uma única pesquisa, mais de um instrumento poderia ser utilizado.

A utilização dos instrumentos é um dado relevante para a análise dessa pesquisa, pois demonstra como o construto é avaliado no contexto educacional. Atualmente, na avaliação do autoconceito no contexto escolar e educacional, poucos instrumentos têm sido empregados, e os testes, prerrogativa dos psicólogos, nem sempre são utilizados. Ao lado disso, deve-se considerar que, entre os instrumentos utilizados, o único que consta na lista de testes aprovados para o uso profissional do Sistema de Avaliação de Testes Psicológicos (SATEPSI, 2007) é a Escala de Autoconceito Infanto-Juvenil (EAC-IJ), portanto, o único que apresenta parâmetros psicométricos adequados para utilização, por psicólogos, em contextos específicos no Brasil. Nesse contexto, é 
válido ressaltar que, para fins de pesquisa, os testes não precisam estar aprovados pelo SATEPSI (2007), pois esses estudos visam ao seu aprimoramento e validação.

Considerando tais resultados, fica evidente a necessidade de pesquisas que focalizem a construção e o desenvolvimento de algum tipo de medida do autoconceito, especialmente daquelas já disponibilizadas nas produções científicas do País. Nesse sentido, a resolução CFP n.o 002/2003 ainda destaca que tais estudos podem ser realizados independentemente da aprovação dos instrumentos no SATEPSI.

Os resultados aqui obtidos também podem estar relacionados às limitações dos estudos desenvolvidos com instrumentos, conforme apontado por vários autores, entre os quais SouzaFilho et al. (2006). Em sua investigação, os autores destacaram que as pesquisas no campo dos instrumentos psicológicos ainda se mostram restritas e desenvolvidas em sua maior parte no meio acadêmico, e alertam, ainda, para a falta de estudos referentes aos parâmetros psicométricos desses instrumentos.

Deve-se ressaltar a proporção ínfima em que os testes foram utilizados para a avaliação do autoconceito. De fato, dos 10 artigos avaliados, somente em 6 foram utilizados testes. Os dados relacionados às referências utilizadas na fundamentação dos artigos avaliados são apresentados na seqüência.

\section{Referências utilizadas}

Para a análise da freqüência das referências utilizadas na fundamentação dos artigos avaliados, foram selecionadas nove categorias: capítulo de livros, livros, trabalhos apresentados em congressos, trabalhos de conclusão de curso, dissertações, teses, artigos, testes e outros. Esses resultados podem ser visualizados na Tabela 2.

Tabela 2. Freqüências das referências utilizadas na fundamentação dos artigos pesquisados.

\begin{tabular}{llll}
\hline Referências & Tipo & $F$ & $\%$ \\
\hline Capítulos de livros & Nacional & 17 & 4,91 \\
& Internacional & 23 & 6,65 \\
Livros & Total & 40 & 11,56 \\
& Nacional & 22 & 6,36 \\
& Internacional & 50 & 14,50 \\
Congressos & Total & 72 & 20,86 \\
& Nacional & 5 & 1,45 \\
& Internacional & 1 & 0,29 \\
Artigos & Total & 6 & 1,74 \\
& Nacional & 51 & 14,70 \\
& Internacional & 139 & 40,20 \\
Dissertações & Total & 190 & 54,90 \\
Teses & ---------------- & 11 & 3,18 \\
Testes & --------------- & 7 & 2,02 \\
Outros* & -------------- & 10 & 2,89 \\
Total & ------------- & 10 & 2,89 \\
\hline
\end{tabular}


De acordo com os dados dispostos na Tabela, pode-se destacar que os artigos de periódicos foram as fontes mais consultadas. Após os artigos científicos, as citações mais freqüentes dizem respeito a livros e capítulos de livros, sendo que a literatura internacional é consideravelmente mais citada que a nacional. Esse fato pode ser entendido à luz do maior desenvolvimento, no exterior, de pesquisas relativas à avaliação do autoconceito no contexto educacional. Um aspecto interessante é o fato de a literatura nacional, embora restrita (nesta pesquisa, trabalhou-se com apenas 10 artigos), evidenciar uma grande quantidade de citações de pesquisas nacionais, embora em menor quantidade que as citações estrangeiras.

\section{À guisa de conclusão}

Nesta pesquisa, objetivou-se realizar um levantamento dos trabalhos publicados nos últimos dez anos em periódicos científicos referentes à avaliação do autoconceito no contexto escolar. Para tanto, foram consideradas algumas categorias, quais sejam, autoria, Região em que prevaleceram as pesquisas, instituição de filiação, tipo de população pesquisada, instrumentos utilizados na avaliação do autoconceito e referências utilizadas na fundamentação dos artigos. Dessa forma, considera-se que devem ser realizadas pesquisas com maior aprofundamento sobre o construto.

Discussões sobre as amostras estudadas também merecem destaque, uma vez que a maior parte das pesquisas analisadas foi realizada com crianças. Essa questão pode ser foco de futuras investigações que, mais do que "diagnosticar" de forma isolada grupos de crianças, avaliem o autoconceito no âmbito escolar de forma eficiente, o que possibilitaria a compreensão da importância da escola na aquisição e no desenvolvimento do autoconceito do aluno, seja ele criança, adolescente ou adulto.

Sugerem-se, ainda, estudos acerca da construção e do desenvolvimento de instrumentos adequados para a avaliação do autoconceito no contexto escolar, uma vez que os resultados aqui obtidos demonstraram que ela nem sempre tem sido realizada com base em instrumentos ou técnicas confiáveis e que sejam realmente sensíveis para a captação das diferentes variáveis que fazem deste um construto multideterminado. Ao lado disso, ressalta-se que o desenvolvimento de outras pesquisas como esta possibilitariam não apenas a superação das carências detectadas e a criação de novas medidas, mas também o incremento do desempenho acadêmico desses alunos por meio da implantação de programas de apoio aos indivíduos que apresentem prejuízos nessa área.

Nesse sentido, os resultados desta pesquisa contribuem tanto com a área escolar e educacional quanto com os estudos que se prestam à avaliação da produção científica nacional, pois, apesar de um aumento das investigações bibliométricas, novos estudos são necessários, a fim de se possibilitar o desenvolvimento e a compreensão dos construtos ou de sua avaliação, quaisquer que sejam. Do mesmo modo, devem-se considerar as limitações deste estudo, que focalizou apenas periódicos classificados como Nacional A, ou seja, as publicações de qualidade reconhecida, e se restringiu à análise descritiva dos dados obtidos. 
Adriana Cristina Boulhoça Suehiro*

Psicóloga, Doutora em Psicologia pela Universidade São Francisco/Itatiba.

Docente da Universidade Federal do Recôncavo da Bahia.

\section{Fabián Javier Marín Rueda}

Psicólogo, Doutor em Avaliação Psicológica pela Universidade São Francisco/Itatiba.

Evelin Zago de Oliveira

Psicóloga pela Universidade São Francisco/Itatiba.

\section{Silvia Verônica Pacanaro}

Psicóloga, Mestre em Avaliação Psicológica pela Universidade São Francisco/Itatiba.

*Endereço para envio de correspondência:

Rua Avenida Ipê Amarelo, 820 - Casa 80

13175-667 - Villa Flora Sumaré- SP

E-mail: dricbs@yahoo.com.br

Recebido 26/10/2007; Reformulado 28/07/2008 ; Aprovado 29/07/2008 . 


\section{Referências}

Antunes, R. (1998). Adeus ao trabalho: ensaio sobre as metamorfoses e a centralidade do mundo do trabalho (5a ed.). São Paulo: Cortez; Campinas, SP: Editora da UNICAMP.

Antunes, R. (1999). Os sentidos do trabalho: ensaio sobre a afirmação e a negação do trabalho. São Paulo: Boitempo Editorial.

Caldana, A. C. F. (2000). Desemprego e qualidade de vida: estratégias de inclusão social e sobrevivência. Dissertação de Mestrado em Psicologia, Faculdade de Filosofia, Ciências e Letras de Ribeirão Preto, Universidade de São Paulo, Ribeirão Preto, SP.

Castells, M. (2003). A sociedade em rede (7a ed., V. Majer \& K. B. Gerhardt, trads.). São Paulo: Paz e Terra.

Demo, P. (2001). Brincando de solidariedade: política social de primeira dama. In M. O. S. Silva (Coord.). O comunidade solidária: o não enfrentamento da pobreza no Brasil (pp. 4369). São Paulo: Cortez.

Demo, P. (2002). Introdução à sociologia: complexidade, interdisciplinaridade e desigualdade social. São Paulo: Atlas.

Guanaes, C. (2004). A terapia de grupo como recurso conversacional: o processo de negociação de sentidos em um grupo ambulatorial de curta duração em saúde mental. Tese de Doutorado em Psicologia, Faculdade de Filosofia, Ciências e Letras de Ribeirão Preto, Universidade de São Paulo, Ribeirão Preto, SP.

Jovchelovitch, S. (2000). Representações sociais e esfera pública: a construção simbólica dos espaços públicos no Brasil. Petrópolis, RJ: Vozes.

Landim, L., \& Scalon, M. C. (2001). Quem dá e quem não dá - eis a questão. RETS - Revista do Terceiro Setor. Recuperado em 30 de abril de 2001, de http://www.rits.org.br

Lyet, P. (1998). The organization of volunteers a challenge for actors in the social field. Revue du MAUSS, (11), 280-294.
Lukács, G. (1974). História e consciência de classe: estudos de dialética marxista. Porto: Publicações Escorpião.

Mead, G. H. (1934). Mind, self and society: Social attitudes and the physical world. Chicago: University of Chicago Press. Recuperado em 2 de maio de 2005, de http://www2.pfeiffer. edu/ /ridener/DSS/Mead/MINDSELF.HTML

Mészáros, I. (2002). Para além do capital: rumo a uma teoria da transição (P. C. Castanheira \& S. Lessa, trads.). São Paulo: Boitempo Editorial.

Minayo, M. C. S., \& Deslandes S. L. (2002). (Orgs.). Caminhos do pensamento: epistemologia e método. Rio de Janeiro: Fiocruz.

Pochmann, M. (1999). O trabalho sob fogo cruzado. São Paulo: Contexto.

Salamon, L. (1997). Estratégias para o fortalecimento do terceiro setor. In E. B. loschpe, Terceiro setor: desenvolvimento social sustentado (pp. 89-112). Rio de Janeiro: Paz e Terra.

Selli, L., \& Garrafa, V. (2005). Bioética, solidariedade crítica e voluntariado orgânico. Revista de Saúde Pública, 39(3), 473-478.

Spink, M. J. P. (1995). O conhecimento no cotidiano: as representações sociais na perspectiva da psicologia social. São Paulo: Brasiliense.

Spink, M. J. P. (Org.). (1999). Práticas discursivas e produção de sentidos no cotidiano: aproximações teóricas e metodológicas. São Paulo: Cortez.

Vigotski, L. S. (1998). A formação social da mente: o desenvolvimento dos processos psicológicos superiores (6a ed., J. Cipolla Neto, L. S. M. Barreto, \& S. C. Afeche, trads.). São Paulo: Martins Fontes. (Trabalho original publicado em 1930-1936) 